\title{
THE SPECTRAL SCALE AND THE $k$-NUMERICAL RANGE
}

\author{
CHARLES A. AKEMANN \\ Department of Mathematics, University of California, Santa Barbara, California 93106-3038, U.S.A. \\ e-mail: akemann@math.ucsb.edu \\ and JOEL ANDERSON* \\ Department of Mathematics, Pennsylvania State University, University Park, \\ Pennsylvania 16802-6401, U.S.A. \\ e-mail: anderson@math.psu.edu
}

(Received 19 December, 2001; accepted May, 2003)

\begin{abstract}
Suppose that $c$ is a linear operator acting on an $n$-dimensional complex Hilbert Space $H$, and let $\tau$ denote the normalized trace on $B(H)$. Set $b_{1}=\left(c+c^{*}\right) / 2$ and $b_{2}=\left(c-c^{*}\right) / 2 i$, and write $B$ for the spectral scale of $\left\{b_{1}, b_{2}\right\}$ with respect to $\tau$. We show that $B$ contains full information about $W_{k}(c)$, the $k$-numerical range of $c$ for each $k=1, \ldots, n$. This is in addition to the matrix pencil information that has been described in previous papers. Thus both types of information are contained in the geometry of a single 3-dimensional compact, convex set. We then use spectral scales to prove a new fact about $W_{k}(c)$. We show in Theorem 3.4 that the point $\lambda$ is a singular point on the boundary of $W_{k}(c)$ if and only if $\lambda$ is an isolated extreme point of $W_{k}(c)$ : i.e. it is the end point of two line segments on the boundary of $W_{k}(c)$. In this case $\lambda=(n / k) \tau(c z)$, where $z$ is a central projection in the algebra generated by $c$ and the identity. In addition we show how the general theory of the spectral scale may be used to derive some other known properties of the $k$-numerical range.
\end{abstract}

2000 Mathematics Subject Classification. 15A60.

0. Introduction and notation. The spectral scale was introduced by the present authors and Nik Weaver in [1] and further developed by the authors in [2]. It is defined for any finite set of self-adjoint operators in a finite von Neumann algebra. The main theme in [1] and [2] is that full spectral information about real linear combinations of such operators is contained in the spectral scale and that much of this information is reflected by the geometry of the spectral scale.

In the present paper we view the spectral scale from another perspective and thereby show that full information about the $k$-numerical range of a finite dimensional operator is also contained in its spectral scale. In fact it turns out that certain crosssections of the spectral scale (which we call isotrace slices) are isomorphic to the $k$-numerical ranges via an affine transformation. Thus, in this restricted case at least, two rather different sets of data are combined into one three dimensional, compact, convex set. Although we restrict consideration in this paper to the finite dimensional world, we shall write this paper using the language of operators (rather than matrices)

*The second author was partially supported by the National Science Foundation during the period of research that resulted in this paper. 
to emphasize how easily many of the concepts generalize to infinite dimensional situations. Indeed, results analogous to those presented here also hold in the infinite dimensional case. These will be presented in a forthcoming paper.

In what follows we shall show that many of the known properties of the $k-$ numerical range may be easily derived from the general theory of the spectral scale. In addition some new results will be derived. These are presented in Section 3 below. We are indebted to Professor Chi-Kwong Li for pointing out several references that we had overlooked.

The notation developed here will be used throughout the rest of the paper. Let $c$ denote a linear operator on an $n$-dimensional complex Hilbert space $H$ and let $\tau$ denote the normalized trace on $B(H)$, the algebra of all linear operators on $H$. Write $N$ for the subalgebra of $B(H)$ generated by $c, c^{*}$ and the identity 1 of $B(H)$. Set $b_{1}=\left(c+c^{*}\right) / 2$ and $b_{2}=\left(c-c^{*}\right) / 2 i$. The spectral scale was defined in [1] and [2] via the map $\Psi$ given by the formula

$$
\Psi(a)=\left(\tau(a), \tau\left(b_{1} a\right), \tau\left(b_{2} a\right)\right),
$$

and the spectral scale $B=B\left(b_{1}, b_{2}\right)$ was defined as

$$
B=\{\Psi(a): a \in B(H), 0 \leq a \leq 1\} .
$$

It is convenient for the present paper to view the second and third real coordinates as a single complex number. The definition of $\Psi$ then becomes

$$
\Psi(a)=(\tau(a), \tau(c a))
$$

and we now define the spectral scale $B=B(c)$ of $c$ by the formula

$$
B=\{\Psi(a): a \in B(H), 0 \leq a \leq 1\} .
$$

Thus, we now view $B$ as a subset of $\mathbb{R} \times \mathbb{C}$, rather than as a subset of $\mathbb{R}^{3}$. As shown in [1, Theorem 2.4], $B=\Psi\left(N_{1}^{+}\right)$, where for any self-adjoint subalgebra $M$ of $B(H)$,

$$
M_{1}^{+}=\{a \in M: 0 \leq a \leq 1\} .
$$

As noted above, the geometry of the spectral scale reflects spectral data for real linear combinations of $b_{1}, b_{2}$; i.e. matrix pencil information. Information of this sort is widely valued as documented in [9]. The basis for this current work is the observation that $B$ also essentially contains the $k$-numerical range of $c$ for each $1 \leq k \leq n$.

Recall that the numerical range of $c$ is by definition

$$
W(c)=\{\langle c \mathbf{x}, \mathbf{x}\rangle: \mathbf{x} \in H \text { and }\|\mathbf{x}\|=1\} .
$$

We use $\sigma(c)$ to denote the spectrum of $c$.

Observe that if $W(c)$ is a line segment, then it follows from [7, §1.2] that the endpoints of $W(c)$ are reducing eigenvalues of $c$. Also, $W(c)=\{\lambda\}$ if and only if $c=\lambda 1$. Since these cases are not of interest to us, we shall restrict our considerations to the case in which $W(c)$ is a two dimensional set.

The numerical range has a corner at $\lambda$ if $W(c)$ has dimension two and there is more than one tangent line of support for $W(c)$ at $\lambda$. The presence of a corner in the numerical range signals the fact that $c$ enjoys a special structure. Further, it turns out that corners in $W(c)$ must be lineal in the sense that there are two tangent lines of 
support that intersect the boundary in line segments of positive length. Since we shall show that an analogous result hold for the $k$-numerical range, we now discuss this in more detail. Recall that a point on the boundary of a convex subset of $\mathbb{R}^{2}$ is said to be singular if the boundary curve is not differentiable at this point.

THEOREM 0.1. If $W(c)$ has dimension two and the boundary of $W(c)$ is singular at $\lambda$, then the following statements hold.

(1) $W($ c) has a corner at $\lambda$.

(2) $\lambda$ is a reducing eigenvalue for $c$.

(3) $\lambda$ is lineal.

Proof. Let us begin by presenting a proof of the assertion in (1). Translating and rotating if necessary, we may assume that $\lambda=0, W(c)$ lies in the upper half plane and that the positive imaginary axis intersects the interior of $W(c)$. In this case we can find a convex function $f$ defined on an open interval containing 0 and whose graph gives a portion of the boundary of $W(c)$ that contains 0 . Since 0 is a singular point of the boundary, $f$ is not differentiable at 0 and since $f$ is convex, it follows that

$$
f_{-}^{\prime}(0)=\lim _{h \rightarrow 0^{-}} \frac{f(h))}{h}<\lim _{h \rightarrow 0^{+}} \frac{f(h))}{h}=f_{+}^{\prime}(0) .
$$

Thus, the lines through 0 with slopes $f_{ \pm}^{\prime}(0)$ are tangent to $W(c)$.

Kippenhahn established assertion (2) in [8, Satz 13]. The proof offered below seems to be new. It is convenient to rotate once more so that $\lambda=0$ and $W(c)$ lies in the right half plane so that the corner has the form shown below, where both $L_{i}$ are tangent to $W(c)$ at $0, L_{1}$ has positive slope and $L_{2}$ has negative slope, as shown below.

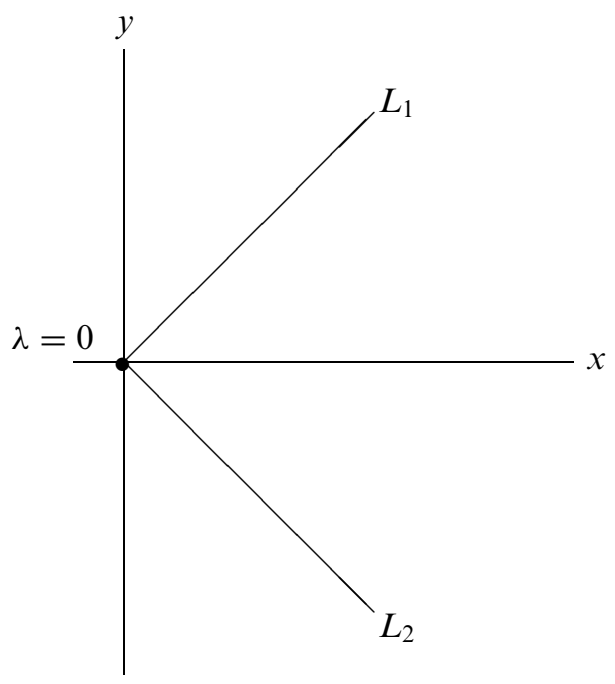

Since $W(c)$ is contained in the right half plane, we get $b_{1} \geq 0$ and, since there is a unit vector $\mathbf{x}$ such that $\left\langle b_{1} \mathbf{x}, \mathbf{x}\right\rangle=0$, we get $b_{1} \mathbf{x}=0$, by the remark in the second paragraph preceding Theorem 0.1 .

We may now select $\theta \neq 0$ so small that $W\left(e^{i \theta} c\right)$ is also contained in the right half plane. Arguing as above, we get that

$$
\operatorname{Re}\left(e^{i \theta} c\right) \mathbf{x}=\left(\cos \theta b_{1}-\sin \theta b_{2}\right) \mathbf{x}=0
$$


and so $b_{2} \mathbf{x}=0$. Therefore $b_{1} \mathbf{x}=b_{2} \mathbf{x}=c \mathbf{x}=0$. Hence, $\lambda$ must be a reducing eigenvalue for $c$.

Assertion (3) is due to Lancaster [10, Corollary 4].

Observe that the proof above shows that if $C$ is any compact, convex, two dimensional subset of $\mathbb{R}^{2}$, then the boundary of $C$ is singular at $\lambda$ if and only if $C$ has a corner at $\lambda$.

The $k$-numerical range of $c$ is defined by the formula

$$
W_{k}(c)=\left\{\frac{1}{k} \sum_{i=1}^{k}\left\langle c \mathbf{x}_{i}, \mathbf{x}_{i}\right\rangle: \text { the } \mathbf{x}_{i} \text { 's are orthonormal }\right\} \quad(1 \leq k \leq n) .
$$

An easily verified alternative definition is as follows:

$$
W_{k}(c)=\left\{\frac{n}{k} \tau(c p): p \text { is a projection of rank } k\right\} .
$$

Observe that when $k=n$ we have

$$
W_{n}(c)=\tau(c),
$$

so that $W_{n}(c)$ consists of a single point. As the results in this paper make clear it is natural to include the case in which $k=0$ and to define $W_{0}(c)=0$. Since we have $W_{1}(c)=W(c)$, this notion is a generalization of the standard numerical range. Let us now review some of the basic properties of the $k$-numerical range.

THEOREM 0.2. If $1 \leq k \leq n$, then the following statements hold.

(1) $W_{k}(c)$ is a compact, convex subset of $\mathbb{C}$.

(2) If $1 \leq k \leq n$ and $p$ denotes any projection of rank $k$, then

$$
k W_{k}(c)=\left\{n \tau\left(u p u^{*} v c v^{*}\right): u \text { and } v \text { are unitary }\right\} .
$$

(3) We have $k W_{k}(c)=n \tau(c)-(n-k) W_{n-k}(c)$.

(4) The operator $c$ is normal if and only if $W_{k}(c)$ is a polygon for each $k$.

(5) The operator $c$ is normal if and only if $W_{[n / 2]}$ is a polygon.

(6) If $W_{k}(c)$ has a corner at $\lambda$, then $c=c_{1} \oplus c_{2}$, where $c_{1}$ has dimension $k$ and $k \lambda=n \tau\left(c_{1}\right)$.

Proof. The assertion in (1) is due to Berger who introduced the $k$-numerical range in his thesis [3]. A proof may be found in [5, Problem 167]. The assertion in (2) follows easily from the fact that $\tau$ is central and the definition of $W_{k}(c)$. Assertion (3) follows from the fact that $\tau(c p)+\tau(c(1-p))=\tau(c)$. Assertion (4) is proved in [12, Theorem 3]. The remarkable result in assertion (5) is due to Li, Sung and Tsing [11] and the assertion in (6) was first proved by Marcus and Filippenko in [13].

Let us now describe our results in more detail. The key to understanding the role played by the $k$-numerical range in the spectral scale is the notion of an isotrace slice of the spectral scale. If $0 \leq t \leq 1$, then the isotrace slice of $B$ at $t$ is by definition

$$
I_{t}=\left\{\mathbf{x}=\left(x_{0}, z\right) \in B: x_{0}=t\right\}
$$


We prove in Theorem 1.3 that if $0<k<n$ and we define the map $\pi_{k}$ from $\mathbb{C}$ to $\mathbb{R} \times \mathbb{C}$ by $\pi_{k}(z)=(k / n, k z / n)$, then $\pi_{k}$ is an affine map that is a bijection from $W_{k}(c)$ onto $I_{k / n}$. Thus, we may view the $k$-numerical range as a subset of the spectral scale.

It is also shown in Section 1 (Theorem 1.1) that the extreme points of $B$ lie on the isotrace slices of the form $I_{k / n}$ for $k=0,1, \ldots, n$ and so $B$ is the convex hull of this finite collection of sets. In Section 2 we present some examples (and pictures) of various spectral scales.

It turns out that the general theory of spectral scales that was developed in [1] and [2] may be used to derive the assertions (4) and (6) in Theorem 0.2. In fact, assertion (4) follows immediately from the identification of $W_{k}(c)$ with the isotrace slice $I_{k / n}$ and [2, Corollary 5.6]. Assertion (6) is proved below in Theorem 3.4. In addition we show that a singularity on the boundary of $W_{k}(c)$ always occurs at an isolated extreme point of $W_{k}(c)$. Such a point is obviously lineal. Furthermore, such points correspond to central projections in the algebra $N$.

As our results show, the spectral scale provides a new way to study $n$-tuples of self-adjoint finite dimensional operators. In the case under study here, when there are just two operators so that $B$ is a subset of three dimensional real euclidean space (or $\mathbb{R} \times \mathbb{C}$ ), we may actually visualize $B$ as shown in the examples and pictures in Section 2. These pictures were created using a MATLB program written by Jeff Duzak as part of an REU research project supervised by the second author. Readers can contact the second author for a copy of this program which is quite useful for testing conjectures. This program also draws isotrace slices of $B$ and so, by the results of this paper, it also draws scale models of $k$-numerical ranges.

1. Isotraces and extreme points. The spectral scale has a striking structure in the finite dimensional case under consideration here that we describe in the next Theorem.

THEOREM 1.1. If $\mathbf{x}$ is an extreme point of $B$, then it lies in an isotrace slice of the form $I_{k / n}$, where $k=0,1, \ldots, n$.

Proof. Since $\mathbf{x}$ is an extreme point of $B$, it has the form $\Psi(p)$, where $p$ is a projection in $N$ by [1, Theorem 2.3(1)]. If $p$ has rank $k$, then $\tau(p)=k / n$ and so $\Psi(p)=(k / n, s)$, where $s=\tau(c p)$. Hence, $\mathbf{x} \in I_{k / n}$.

For example, if $n=3$, then extreme points of $B$ come from projections of trace $0,1 / 3,2 / 3$ or 1 . While $I_{0}$ and $I_{1}$ are always the single points 0 and $\Psi(1)$, in generic examples $I_{1 / 3}$ and $I_{2 / 3}$ are solid ellipses whose boundaries consist of extreme points of $B$. Hence, generically, the boundary of $B$ between two successive isotrace slices consists of one dimensional faces. The boundary between $I_{0}$ and $I_{1 / 3}$ is typically a skewed cone and, by the symmetry of $B$, the same is true for the boundary of $B$ between $I_{2 / 3}$ and $I_{1}$. However, as one can see in Examples 2.2 and 2.3 below, $B$ may have planar faces. A complete description and interpretation of the faces of $B$ may be found in [2, §3]. Further, in [2, Corollary 5.6] we showed that $N$ is abelian and finite-dimensional if and only if the spectral scale has a finite number of extreme points. Thus, in finite dimensions, one can "see" that $N$ is abelian from the shape of $B$.

We now show how the $k$-numerical range may be identified with the isotrace slice $I_{k / n}$. This identification depends on a simple convexity result, which we now present. If $0<t<1$ and $M$ is any self-adjoint subalgebra of $B(H)$, then we write

$$
M_{1, t}^{+}=\left\{a \in M_{1}^{+}: \tau(a)=t\right\} .
$$


LEMMA 1.2. The extreme points of $B(H)_{1, k / n}^{+}$are precisely the projections of rank $k$.

Proof. If $p$ is a projection of rank $k$, then it is an extreme point of $B(H)_{1}^{+}$and so it is also an extreme point of $B(H)_{1, k / n}^{+}$. For the converse, suppose that $a$ is in $N_{1, k / n}^{+}$, but $a$ is not a projection. Write $\alpha_{1}, \ldots, \alpha_{n}$ for the eigenvalues of $a$. Since $\tau(a)=k / n$ we get that $\alpha_{1}+\cdots+\alpha_{n}=k$ and, since $a$ is not a projection, we have $0<\alpha_{i}<1$, for at least one index $i$. Since $\alpha_{1}+\cdots+\alpha_{n}=k$, there must also be an index $j \neq i$ such that $0<\alpha_{j}<1$. Relabeling if necessary, we may assume that $0<\alpha_{1} \leq \alpha_{2}<1$.

Since $\alpha_{1}+\alpha_{2}-1<\alpha_{1}$ and $0<\alpha_{1}$ we may select $\lambda$ and $\gamma$ such that

$$
\max \left\{0, \alpha_{1}+\alpha_{2}-1\right\}<\lambda<\alpha_{1} \text { and } \alpha_{2}<\gamma<\min \left\{1, \alpha_{1}+\alpha_{2}\right\}
$$

Now write

$a_{1}=\left[\begin{array}{ccccc}\lambda & 0 & 0 & \ldots & 0 \\ 0 & \alpha_{2}+\alpha_{1}-\lambda & 0 & \ldots & 0 \\ 0 & 0 & \alpha_{3} & \ldots & 0 \\ \vdots & \vdots & \vdots & \ddots & \vdots \\ 0 & 0 & 0 & \ldots & \alpha_{n}\end{array}\right]$ and $a_{2}=\left[\begin{array}{ccccc}\gamma & 0 & 0 & \ldots & 0 \\ 0 & \alpha_{2}+\alpha_{1}-\gamma & 0 & \ldots & 0 \\ 0 & 0 & \alpha_{3} & \ldots & 0 \\ \vdots & \vdots & \vdots & \ddots & \vdots \\ 0 & 0 & 0 & \ldots & \alpha_{n}\end{array}\right]$.

Observe that $a_{1} \neq a_{2}$ because $\lambda<\alpha_{1} \leq \alpha_{2}<\gamma$. Since the diagonal entries of $a_{1}$ and $a_{2}$ lie in $[0,1]$ and sum to $k$, these matrices are elements of $B(H)_{1, k / n}^{+}$. As $\lambda<\alpha_{1}<\gamma$ there is a real number $t$ with $0<t<1$, such that $\alpha_{1}=t \lambda+(1-t) \gamma$. Next, note that

$$
\begin{aligned}
t\left(\alpha_{1}+\alpha_{2}-\lambda\right)+(1-t)\left(\alpha_{1}+\alpha_{2}+\gamma\right) & =\alpha_{1}+\alpha_{2}-(t \lambda+(1-t) \gamma) \\
& =\alpha_{1}+\alpha_{2}-\alpha_{1}=\alpha_{2}
\end{aligned}
$$

and therefore $a=t a_{1}+(1-t) a_{2}$. Thus $a$ is not an extreme point.

THEOREM 1.3. If $0<k<n$ and we define the map $\pi_{k}$ of $\mathbb{C}$ into $\mathbb{R} \times \mathbb{C}$ by the formula.

$$
\pi_{k}(\lambda)=(k / n, k \lambda / n)
$$

then $\pi_{k}$ is an affine bijection from $W_{k}(c)$ onto $I_{k / n}$.

Proof. If $\lambda \in W_{k}(c)$, then there is a projection $p$ with rank $k$ such that $k \lambda=n \tau(p c)$ and the point $(\tau(p), \tau(p c))$ is in $I_{k / n}$ because $\tau(p)=k / n$. Thus,

$$
\pi_{k}(\lambda)=(k / n, k \lambda / n)=(k / n, \tau(p c)) \in I_{k / n} .
$$

Hence $\pi_{k}$ maps $W_{k}(c)$ into $I_{k / n}$ and $\pi_{k}$ is clearly a one-to-one map.

Now suppose that $(k / n, \lambda) \in I_{k / n}$ so that $\lambda=\tau(c a)$, for some $a \in N_{1, k / n}^{+}$. By Lemma 1.2, the Krein-Milman Theorem [16, Theorem 2.6.16 ] and [14, Theorem 17.1], $a$ is a convex combination of projections of rank $k$. Since $W_{k}(c)$ is convex by part (1) of Theorem 0.2 and points of the form $(n / k) \tau(c p)$ are in $W_{k}(c)$, we deduce that $(n / k) \lambda \in W_{k}(c)$.

2. Examples. In this section we describe the spectral scale for four examples.

EXAMPLE 2.1. If

$$
c=\left[\begin{array}{cc}
1+\frac{i}{2} & \frac{i}{2} \\
\frac{i}{2} & \frac{i}{2}
\end{array}\right],
$$


then the nontrivial projections in $N$ must have trace $1 / 2$ and so $I_{1 / 2}$ is the only isotrace slice of $B$ that contains nontrivial extreme points. It turns out that $I_{1 / 2}$ is the disk of radius $1 / 4$ centered at $(1 / 2,(1+i) / 4)$. Since

$$
B=\operatorname{conv}\left(0, I_{1 / 2},(1,(1+i) / 2)\right)
$$

the spectral scale in this case is a pair of skewed circular cones joined at their bases as shown below.

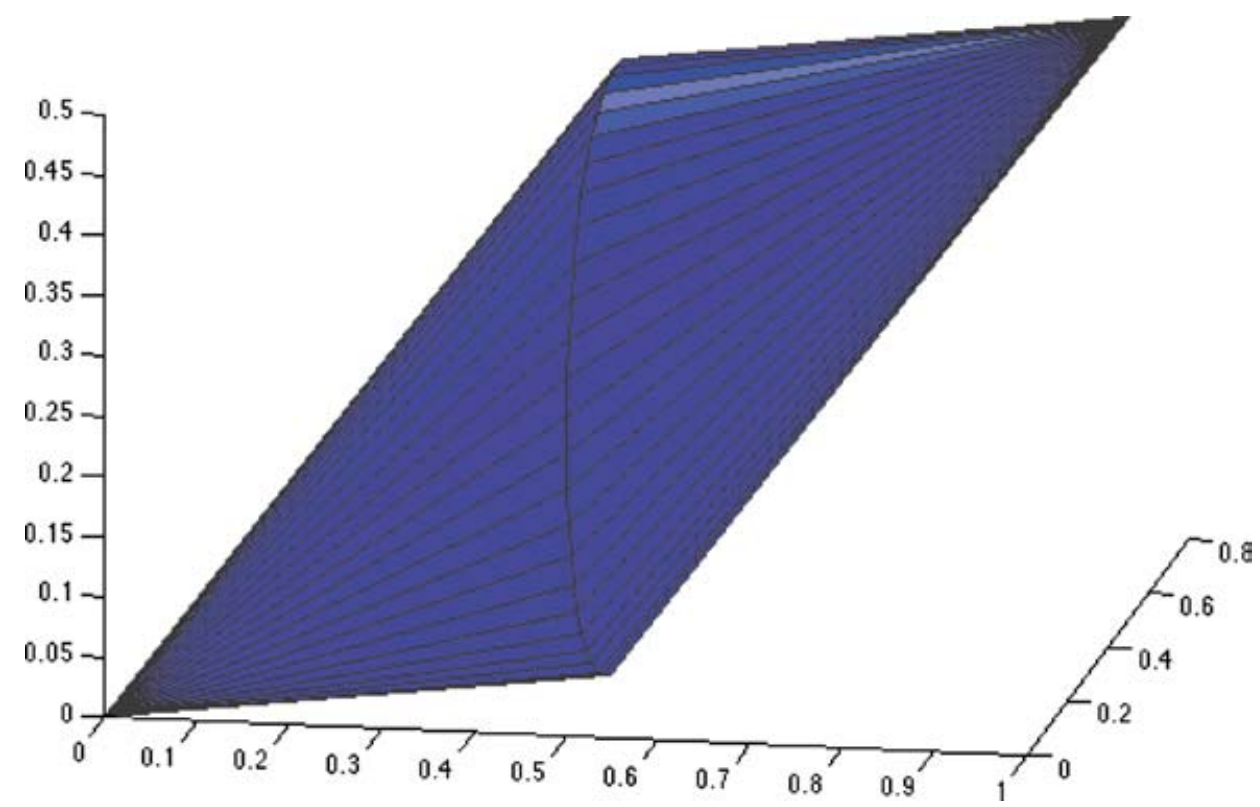

In this case the extreme points of $B$ are $0, \Psi(1)$ and points on the circular boundary of $I_{1 / 2}$. The one dimensional faces are the line segments joining 0 and $\Psi(1)$ to the extreme points on the circle. There are no faces of dimension two.

We now present a $3 \times 3$ example in which the spectral scale has a "flat spot".

EXAMPLE 2.2. Write

$b_{1}=\left[\begin{array}{lll}1 & 0 & 1 \\ 0 & 2 & 1 \\ 1 & 1 & 3\end{array}\right], \quad b_{2}=\left[\begin{array}{lll}1 & 0 & 0 \\ 0 & 1 & 0 \\ 0 & 0 & 0\end{array}\right] \quad$ and $\quad c=b_{1}+i b_{2}=\left[\begin{array}{ccc}1+i & 0 & 1 \\ 0 & 2+i & 1 \\ 1 & 1 & 3\end{array}\right]$

We have that $\Psi\left(\left[0, b_{2}\right]\right)$ is a face of $B$ by $\left[1\right.$, Theorem 2.3]. (Here $\left[0, b_{2}\right]=\{a \in N$ : $\left.0 \leq a \leq b_{2}\right\}$.) Furthermore, it follows from the results in $[2, \S 3]$ that this face is two dimensional. In fact, as can be seen from the figure below, it is diamond shaped. 


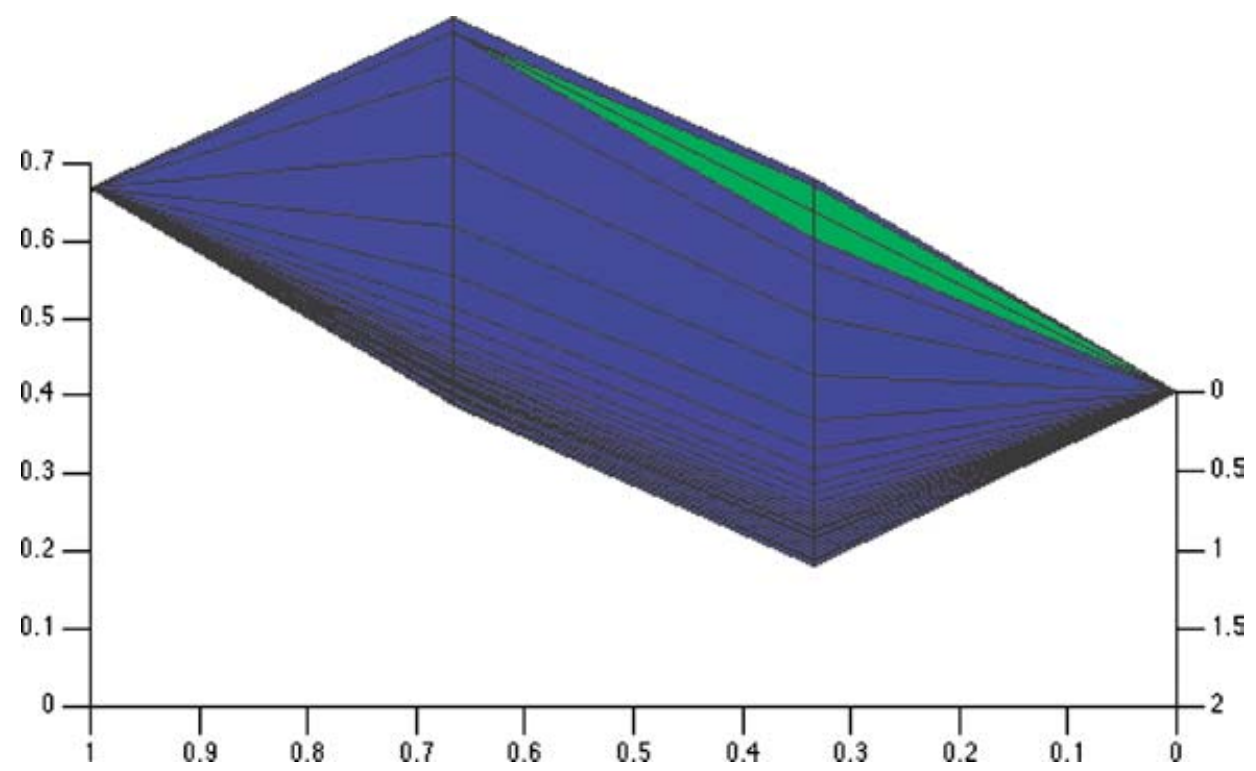

EXAMPLE 2.3. The facial structure of the spectral scale can be quite complex. In the next example there are eight faces of dimension two. If we write

$$
b_{1}=\left[\begin{array}{lllll}
0 & 0 & 0 & 0 & 0 \\
0 & 0 & 0 & 1 & 0 \\
0 & 0 & 0 & 0 & 1 \\
0 & 1 & 0 & 0 & 0 \\
0 & 0 & 1 & 0 & 0
\end{array}\right], \quad b_{2}=\left[\begin{array}{ccccc}
0 & 0 & 0 & 1 & 0 \\
0 & 0 & 0 & 0 & 1 \\
0 & 0 & 0 & 0 & 0 \\
1 & 0 & 0 & 0 & 0 \\
0 & 1 & 0 & 0 & 0
\end{array}\right] \quad \text { and } \quad c=b_{1}+i b_{2}
$$

then the spectral scale is as shown below.

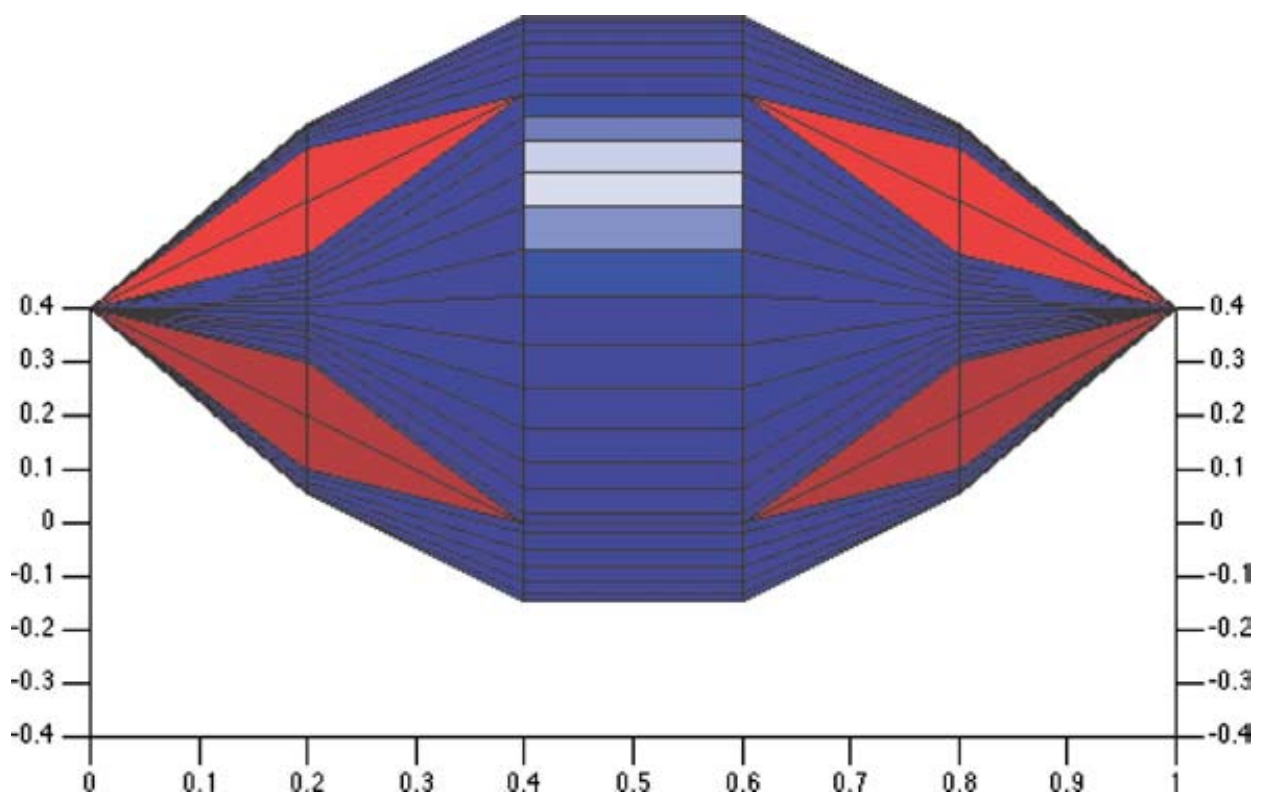


Although it appears from the figure that the lighter portions between the isotrace slices $I_{2 / 5}$ and $I_{3 / 5}$ are two dimensional faces, in fact they consist of faces of dimension one. This anomaly occurred as a result of the MATLAB shading routine employed to draw this picture.

EXAMPLE 2.4. In [1, Example 3.5] we showed that, in the noncommutative case, the spectral scale is not a complete invariant for the operators $b_{1}$ and $b_{2}$ by exhibiting two pairs of inequivalent self-adjoint $3 \times 3$ matrices that shared the same spectral scale. In this example each pair generated the full von Neumann algebra $B(H)$. In our final example we show that the same phenomenon can occur even if one pair is reducible. Write

$$
c_{1}=\left[\begin{array}{ccc}
1+i & 0 & 0 \\
0 & 1+2 i & 1 \\
0 & 1 & 1
\end{array}\right] \text { and } \quad c_{2}=\left[\begin{array}{ccc}
1 & \frac{1}{\sqrt{2}} & 0 \\
\frac{1}{\sqrt{2}} & 1+i & \frac{1}{\sqrt{2}} \\
0 & \frac{1}{\sqrt{2}} & 1+2 i
\end{array}\right]
$$

It is clear that for $c_{2}$ the algebra $N$ is the full algebra $B(H)$, while this is not true for $c_{1}$ because $N$ has non-trivial center.

On the other hand, the associated spectral scales are equal. This follows from the fact that each of these matrices has the same characteristic polynomial and part (2) of [1, Theorem 3.2].

In fact even more is true. Write $S(z)=\operatorname{Re} c_{1}+z \operatorname{Im} c_{1}$ and $T(z)=\operatorname{Re} c_{2}+z \operatorname{Im} c_{2}$. A straightforward calculation shows that

$$
\operatorname{det}(S(z)-w 1)=\operatorname{det}(T(z)-w 1)=\left(-2 z w+2 z+w^{2}-2 w\right)(w-1-z),
$$

so that even if $N$ is irreducible, the associated characteristic polynomial may be reducible.

3. Corners in isotrace slices and the $\boldsymbol{k}$-numerical range. In this section we show that, by using the spectral scale, we can establish results analogous to Theorem 0.1 for the $k$-numerical range. Let us begin by recalling an idea that proved useful in [2]. If $\mathbf{x}$ is an extreme point of a convex set $X$ in $\mathbb{R}^{k}$ which is isolated in the set of all extreme points of $X$, then we say that $\mathbf{x}$ is an isolated extreme point of $X$.

Recall that a face in $B$ is said to be a sharp face if it is contained in at least two hyperplanes of support [2, Definition 4.1]. We showed in [2, Lemma 5.2] that an isolated extreme point of $B$ is always a sharp face. Our main result on corners is Theorem 3.4 below. The proof of this result requires three technical lemmas which we present next. Since the first result is just a slight generalization of Lemma 1.2 and uses the notation from that lemma, the proof is somewhat abbreviated. Recall that if $0<t<1$, then

$$
N_{1, t}^{+}=\left\{a: a \in N_{1}^{+} \text {and } \tau(a)=t\right\} .
$$

LEMMA 3.1. If $a$ is an extreme point of $N_{1, t}^{+}$, then there is a projection $p$ in $N$ such that either $a=p$ or

$$
a=p+\alpha q
$$

where $q$ is a projection in $N$ that is orthogonal to $p, q N q$ has dimension one and $0<\alpha<1$. 
Proof. Fix an extreme point $a$ of $N_{1, t}^{+}$. If $a=p$ is a projection, then the proof is complete. Assume that this is not the case. Let $\alpha_{1}, \ldots, \alpha_{m}$ denote the distinct eigenvalues of $a$ and let $q_{1}, \ldots, q_{k}$ denote the corresponding eigenprojections. Since $a$ is not a projection, there is at least one index $i$ such that $0<\alpha_{i}<1$. If there were another index $j$ with $0<\alpha_{j}<1$, then by adding (resp. subtracting) a small amount to $\alpha_{i}$ and subtracting (resp. adding) a small amount to $\alpha_{j}$ we would get two new elements of $N_{1 t}^{+}$ whose average is $a$, so that $a$ would not not be an extreme point of this set. Hence, $0<\alpha_{i}<1$ and all other indices are 0 or 1 .

If $q_{i} N q_{i}$ did not have dimension one, then it would contain two nonzero orthogonal projections and, arguing as in the previous paragraph, we would again get that $a$ is not an extreme point. Hence, $a$ has the indicated form.

In the following lemma it is convenient to return (briefly) to the picture of $B$ as lying in $\mathbb{R}^{3}$ and then to use some results in [2] that are stated in this framework. If $C$ is a convex subset of $\mathbb{R}^{2}$ with a corner at $\lambda$, then $C$ admits an infinite family of tangent lines of support at $\lambda$ and these lines all lie in a sector of a disk with maximal angle. We call the tangent lines of support that bound this sector the sectorial tangent lines of support. Also, if $z^{ \pm}$are projections in $N$, then we write

$$
\left[z^{-}, z^{+}\right]=\left\{a \in N: z^{-} \leq a \leq z^{+}\right\} .
$$

LEMMA 3.2. If $k$ is an integer with $1 \leq k \leq n, I_{k / n}$ has dimension two and the point $\mathbf{x}=\left(k / n, r_{1}, r_{2}\right)$ lies at a corner on the isotrace slice $I_{k / n}$, then the following statements hold.

(1) There is a sharp face $F$ in $B$ of dimension at most one that contains $\mathbf{x}$.

(2) $F=\Psi\left(\left[z^{-}, z^{+}\right]\right)$, where $z^{ \pm}$are central projections in $N$.

(3) The points $\Psi\left(z^{ \pm}\right)$are isolated extreme points of $B$.

Proof. As $I_{k / n}$ has dimension two and has a corner at $\mathbf{x}$, there are two distinct sectorial tangent lines of support of $I_{k / n}$ in the plane of $I_{k / n}$ at $\mathbf{x}$. Denote these tangent lines by $L_{1}$ and $L_{2}$. Each $L_{i}$ meets $B$ only in the boundary of the isotrace slice $I_{k / n}$ and so they are each disjoint from the interior of $B$. By [16, Corollary 2.4.11], there are distinct planes $P_{1}$ and $P_{2}$ such that $L_{i} \subset P_{i}$ and each $P_{i}$ is disjoint from the interior of $B$. Since each plane contains $\left.\left(k / n, r_{1}, r_{2}\right)\right)$, they are planes of support for $B$. Thus, if we write $F=P_{1} \cap P_{2} \cap B$, then $F$ is a sharp face of $B$.

Since we are now regarding $B$ as a subset of $\mathbb{R}^{3}$ we may use the results in [1] and [2] to get that each plane of support is determined by a spectral pair of the form $(s, \mathbf{t})$, where $s$ is a real number and $\mathbf{t}=\left(t_{1}, t_{2}\right)$ is a nonzero vector in $\mathbb{R}^{2}$. Specifically, by $[\mathbf{1}$, Theorem 2.3] if $P$ is a plane of support for $B$, then there is a spectral pair $(s, \mathbf{t})$ such that points on $P$ satisfy an equation of the form

$$
-s x_{0}+t_{1} x_{1}+t_{2} x_{2}=\alpha
$$

The constant $\alpha$ is determined as follows. We write $b_{\mathbf{t}}=t_{1} b_{1}+t_{2} b_{2}$ and let $p_{s, \mathbf{t}}^{+}$and $p_{s, \mathbf{t}}^{-}$denote the spectral projections of $b_{\mathbf{t}}$ corresponding to the intervals $(-\infty, s]$ and $(-\infty, s)$. With this notation, we have

$$
\alpha=\tau\left(\left(b_{\mathbf{t}}-s 1\right) p_{s, \mathbf{t}}^{ \pm}\right)
$$

Observe that $(-s, \mathbf{t})$ is a normal vector for this plane. 
Since $P_{1}$ and $P_{2}$ are distinct planes of $B$ that have nonempty intersection, their normal vectors are linearly independent, and so there exist linearly independent spectral pairs $\left(s_{1}, \mathbf{t}_{1}\right)$ and $\left(s_{2}, \mathbf{t}_{2}\right)$ such that each $P_{i}$ has the equation

$$
-s_{i} x_{0}+t_{i 1} x_{1}+t_{i 2} x_{2}=\alpha_{i}
$$

where $\mathbf{t}_{i}=\left(t_{i 1}, t_{i 2}\right)$. Since the point $\mathbf{x}=\left(k / n, r_{1}, r_{2}\right)$ lies in each plane, we get

$$
-s_{i}(k / n)+t_{i 1} r_{1}+t_{i 2} r_{2}=\alpha_{i}
$$

Now fix a point $\left(x_{i 0}, x_{i 1}, x_{i 2}\right)$ on the tangent line $L_{i}$ and observe that since $I_{k / n}$ lies in the plane $x_{0}=k / n$, we get $x_{i 0}=k / n$. Since this point also lies in the plane $P_{i}$, we get

$$
-s_{i}(k / n)+t_{i 1} x_{i 1}+t_{i 2} x_{i 2}=\alpha_{i}=-s_{i}(k / n)+t_{i 1} r_{1}+t_{i 2} r_{2}
$$

and so

$$
t_{i 1}\left(x_{i 1}-r_{1}\right)+t_{i 2}\left(x_{i 2}-r_{2}\right)=0 \text {. }
$$

If the vectors $\mathbf{t}_{1}$ and $\mathbf{t}_{2}$ were linearly dependent, then it would follow that the tangent lines $L_{1}$ and $L_{2}$ are parallel. Since these lines intersect at a corner of the isotrace slice $I_{k / n}$, this is impossible and therefore the $\mathbf{t}_{i}$ 's must be linearly independent.

Applying [2, Corollary 4.7], we get that $F$ has the form $\Psi\left(\left[z^{-}, z^{+}\right]\right)$, where $z^{ \pm}$are central projections in $N$. Next, since $F$ is a face in $B$, the end points $\Psi\left(z^{ \pm}\right)$are extreme points of $B$. Since the projections $z^{ \pm}$are central, the points $\Psi\left(z^{ \pm}\right)$are isolated extreme points of $B$ by [2, Theorem 5.4(2)] and so assertions (2) and (3) hold. $\mathbb{R} \times \mathbb{C}$.

Let us now return to our previous notation so that we regard $B$ as a subset of

LEMMA 3.3. If $G=\Psi\left(\left[z^{-}, z^{+}\right]\right)$is any face of $B$ that satisfies statements (1), (2) and (3) of Lemma 3.2 and $(t, \gamma) \in G$, then $(t, \gamma)$ is an isolated extreme point of $I_{t}$.

Proof. Since $G$ is a face, its intersection with $I_{t}$ is a face of $I_{t}$ that consists of the single extreme point $(t, \gamma)$, since $G$ has dimension one and since faces of $B$ are transverse to isotrace slices by [2, Theorem 6.4(1)]. If $(t, z)$ were not an isolated extreme point of $I_{t}$, then there would be a sequence $\left(t, \gamma_{j}\right)$ of extreme points in $I_{t}$ that converges to $(t, \gamma)$. Since the inverse images of extreme points contain extreme points, each of these points would have the form $\Psi\left(p_{j}\right)$ or $\Psi\left(p_{j}+\alpha_{j} q_{j}\right)$, where $p_{j}, q_{j}$ and $\alpha_{j}$ are as described in Lemma 3.1. After passing to a subsequence we may assume that $\left(t, \gamma_{j}\right)=\Psi\left(p_{j}+\alpha_{j} q_{j}\right)$. We now assume that $0<\alpha_{j}<1$ and further that $G$ is one dimensional. The other cases are handled similarly, but somewhat more easily.

Since the dimension of $N$ is finite, the range of the trace on the projections in $N$ is finite and, since $\tau\left(p_{j}+\alpha_{j} q_{j}\right)=t$ for each $j$, the $\alpha_{j}$ 's also form a finite set. Hence, by passing to another subsequence, we may assume that each $\alpha_{j}$ equals $\alpha$ and $0<\alpha<1$. We can now find another subsequence (which we continue to index with $j$ 's) such that $p_{j}$ converges to $p$ and $q_{j}$ converges to $q$ Since $p_{j}$ and $q_{j}$ are orthogonal and $q_{j} N q_{j}$ has dimension one, for each $j$, it follows readily that $p$ and $q$ are orthogonal projections, and $q N q$ has dimension one.

Since $\Psi(p+\alpha q)=(t, \gamma) \in G$ and $G=\Psi\left(\left[z^{-}, z^{+}\right]\right)$, we have $z^{-} \leq p+\alpha q \leq z^{+}$and, since $G$ is one dimensional, we get that $z=z^{+}-z^{-} \neq 0$. Hence, $z b_{i} z$ is a scalar multiple of $z$, for $i=1,2$ by [2, Lemma 3.3(1)]. Since $z$ is central, $z(p+\alpha q)$ is a multiple of $z$. 
Thus, $p+\alpha q=z^{-}+z(p+\alpha q)$ and so this element is central. But, since $0<\alpha<1$, this means that both $p$ and $q$ are central. This is impossible since the central projections are isolated in the set of all projections. Hence, $(t, \gamma)$ must be an isolated extreme point of $I_{t}$.

With these preparations, we may now present the main Theorem of this section. Recall that the map $\pi_{k}$ from $\mathbb{C}$ to $\mathbb{R} \times \mathbb{C}$, defined by

$$
\pi_{k}(z)=(k / n, k z / n),
$$

is an affine map that is a bijection from $W_{k}(c)$ onto $I_{k / n}$, by Theorem 1.3.

THEOREM 3.4. If $W_{k}(c)$ has dimension two and if $0<k<n$, then the following statements are equivalent.

(1) $\lambda$ is a singular point on the boundary of $W_{k}(c)$.

(2) $W_{k}(c)$ has a corner at $\lambda$.

(3) There is a face $F$ of $B$ containing the point $(k / n, k \lambda / n)$ that enjoys the properties described below.

(a) $F$ is a sharp face in $B$ of dimension at most one.

(b) $F=\Psi\left(\left[z^{-}, z^{+}\right]\right)$, where $z^{ \pm}$are central projections.

(c) The points $\Psi\left(z^{ \pm}\right)$are isolated extreme points of $B$.

(d) There are faces $F_{1}$ and $F_{2}$ in $B$ of dimension two such that $F_{1} \cap F_{2}=F$ and if $(t, \gamma) \in F$, then $F_{i} \cap I_{t}$ is a line segment for each $i$. Further, if $L_{1}$ and $L_{2}$ denote the sectorial tangent lines of support to $W_{k}(c)$ at $\lambda$, then their images $\pi_{k}\left(L_{i}\right)$ in $B$ intersect the relative interiors of the $F_{i}$ 's.

(4) $\lambda$ is an isolated extreme point of $W_{k}(c)$.

Proof. As in the proof of (1) in Theorem 0.2 and the remark following it, we have that $(1) \Longrightarrow(2)$ because the boundary of $W_{k}(c)$ is convex.

Now suppose that (2) holds. In this case $\pi_{k}(\lambda)=(k / n, k \lambda / n)$ lies at a corner of the isotrace slice $I_{k / n}$ and so we may apply Lemma 3.2 to get that parts $(a),(b)$ and $(c)$ of part (3) are true.

Hence, for each $(t, \gamma) \in F$, there are faces $F_{t, 1}$ and $F_{t, 2}$ in $B$ of dimension two such that $(t, \gamma) \in F_{t, i}$ by [2, Lemma 6.5(3)] and Lemma 3.3. If $F$ has dimension zero, so that it is a single point, then we must have $F_{t, i}=F_{i}$ and so $(d)$ is true in this case.

Now suppose that $F$ has dimension one, fix $(t, \gamma)$ in the relative interior of $F$ and consider the face $F_{t}=F_{t, 1} \cap F$. If $F_{t}$ were a single point, then it would be an extreme point of $B$, which is impossible because it lies in the relative interior of $F$. Hence $F_{t}$ has dimension one. Since $F_{t}$ is contained in $F$ we must have $F_{t}=F$. Since it is obvious (and straightforward to prove) that three distinct faces of dimension two in $B$ cannot intersect in a face of dimension one, we must have that $F_{t, 1}$ is either $F_{1}$ or $F_{2}$. Hence assertion (d) holds in this case.

Thus, in all cases we get that the faces $F_{i}$ have dimension two. Since such faces are transverse to the isotrace slices of $B$ by [2, Lemma 6.4(1)], each line $\pi_{k}\left(L_{i}\right)$ intersects $F_{i}$ in its relative interior. Thus, (2) $\Longrightarrow(3)$.

Next, if (3) is true, then it is clear that $\lambda$ is an isolated extreme point of $W_{k}(c)$ and so (3) $\Longrightarrow$ (4). If (4) holds, then it is also clear that (1) holds and so these four conditions are equivalent.

REMARK 3.5. (1) Suppose that $\lambda$ lies at a corner of $W_{k}(c)$ and $L_{i}$ are the sectorial tangent lines of support for $W_{k}(c)$ at this point. The faces $F_{1}$ and $F_{2}$ described in part (3) 
of Theorem 3.4 were specifically constructed so that the corresponding lines $\pi_{k}\left(L_{i}\right)$ in $\mathbb{R} \times \mathbb{C}$ intersect the relative interiors of the $F_{i}$ 's. If the sharp face $F$ has dimension one, then it bounds precisely two faces of dimension two in the boundary of $B$. In this situation, we say that the union of the $F_{i}$ 's is a shelf of $B$ and $F$ is the edge of the shelf.

(2) Now suppose that $F_{1} \cap F_{2}=F=\{(k / n, k \lambda / n)\}$ is a point. In this case $(k / n, k \lambda / n)$ is an extreme point of $B$ and so it cannot be an interior point of any face of $B$. Thus, this point is an "end point" of any face that contains it. We say that $(k / n, k \lambda / n)$ is a mountain peak in this case. In contrast to the one dimensional case, there are three possibilities for the local geometry of $B$ near $(k / n, k \lambda / n)$, which are as follows.

(a) Each face of dimension one that ends at $(k / n, k \lambda / n)$ is the edge of a shelf in $B$.

(b) The point $(k / n, k \lambda / n)$ bounds at least one shelf in $B$ and at least one face of dimension one that is not the edge of a shelf.

(c) The point $(k / n, k \lambda / n)$ does not bound a shelf.

If $N$ is abelian so that $B$ has a finite number of extreme points, then $(k / n, k \lambda / n)$ lies at a mountain peak of type (a). Observe that since $F_{1} \cap F_{2}$ is a point, the faces that intersect the lines $\pi_{k}\left(L_{i}\right)$ do not intersect in a shelf, as in remark (1) above. However, their boundaries are edges of shelves formed from one of the $F_{i}$ 's and another two dimensional face of $B$. In most abelian examples the corners of the isotrace slices all lie in shelves and there are no mountain peaks. On the other hand, it is fairly straightforward to construct abelian examples where mountain peaks of type (a) occur and it seems possible that there are noncommutative examples which also display this geometry. Examples also show that mountain peaks of type (b) can occur.

If the boundary of the numerical range of $c$ is nonsingular (i.e., does not contain any corners), then $\Psi(0)$ is a mountain peak of type (c). Further, it follows from the symmetry of $B$ that $\Psi(1)$ is also a mountain peak of type (c) in this case. We have not been able to construct an example of a type (c) mountain peak at any other points of $B$.

(3) If $F$ is any sharp face of dimension one in $B$, then it is the edge of a shelf and if $(k / n, k \lambda / n) \in F$, for some $k$, then $\lambda \in W_{k}(c)$. To see this, it is convenient to view $B$ as a subset of $\mathbb{R}^{3}$ so that we may use the notation developed in [1] and [2]. Observe that, since $F$ is a sharp face of dimension one, there must be linearly independent spectral pairs $\left(s_{i}, \mathbf{t}_{i}\right), i=1,2$, such that the corresponding faces

$$
F_{i}=\Psi\left(\left[p_{s_{i}, \mathbf{t}_{i}}^{-}, p_{s_{i}, \mathbf{t}_{i}}^{-}\right]\right)
$$

have dimension two and contain $F$. Further, it follows that the vectors $\mathbf{t}_{i}$ are linearly independent. Indeed, if they were linearly dependent, then replacing $\left(s_{i}, \mathbf{t}_{i}\right)$ with a multiple of itself if necessary, we could assume that $\mathbf{t}_{1}=\mathbf{t}_{2}$. (Recall that replacing a spectral pair by a multiple of itself leaves the associated face unchanged.) In this case since $\left(s_{1}, \mathbf{t}_{1}\right)$ and $\left(s_{2}, \mathbf{t}_{1}\right)$ are linearly independent, we must have that $s_{1} \neq s_{2}$. But then, since these vectors are each normal vectors for $F$ we would get that $F$ has dimension zero by [2, Corollary 4.9(2)], a contradiction. With this we may repeat the remaining portion of the proof of part (3) of Theorem 3.4 verbatim to get that the assertions in part (3) hold for $F$.

(4) Now suppose that $F=\Psi\left(\left[z^{+}, z^{-}\right]\right)$is the sharp edge of a shelf in $B$ so that $z^{ \pm}$ are central projections in $N$ with $z^{-}<z^{+}$. In this case if we write $z=z^{+}-z^{-}$, then we get that $z b_{i}=\beta_{i} z$, for $i=1,2$, and if we put $\gamma=\beta_{1}+i \beta_{2}$, then $z c=\gamma z$, so that $\gamma$ is a 
reducing eigenvalue of $c$. This number is the "complex slope" of $F$ because

$$
\frac{\tau\left(c z^{+}\right)-\tau\left(c z^{-}\right)}{\tau\left(z^{+}\right)-\tau\left(z^{-}\right)}=\frac{\tau(z c)}{\tau(z)}=\frac{\tau(\gamma z)}{\tau(z)}=\gamma .
$$

We note that if $c$ is normal so that every face of dimension one is the sharp edge of a shelf in $B$ and the complex slopes of these faces lie in the spectrum of $c$, then these complex slopes fill out the spectrum. A proof of this fact will appear as part of a more general result in a forthcoming paper.

(5) If $F=\Psi\left(\left[z^{+}, z^{-}\right]\right)$is the sharp edge of a shelf, as in remark (4), and if the rank of $z=z^{+}-z^{-}$is $r$, then $F$ intersects $r$ adjacent isotrace slices. Thus, in general, the point $(k / n, k \lambda / n)$ may be an interior point of the face $F$. However, if the reducing eigenvalues of $c$ have multiplicity one, then $F$ lies between two adjacent isotrace slices. Also $(k / n, k \lambda / n)$ is one of the end points of $F$ and so is an extreme point of $B$.

\section{REFERENCES}

1. Charles A. Akemann, Joel Anderson and Nik Weaver, A geometric spectral theory for $n$-tuples of self-adjoint operators in finite von Neumann algebras, J. Functional Analysis, 164 (1999), 258-292.

2. Charles A. Akemann and Joel Anderson, A geometric spectral theory for $n$-tuples of self-adjoint operators in finite von Neumann algebras: II, Pacific J. Math., to appear.

3. C.A. Berger, Normal dilations, Ph.D. thesis, Cornell University, (1963).

4. Jacques Dixmier, $C^{*}$ - algebras (North-Holland, 1977).

5. P. Halmos, A Hilbert space problem book (Van Nostrand, 1967).

6. F. Hausdorff, Der Wertevorrat einer Bilinearform, Math. Z. 3 (1919), 314-316.

7. Roger A. Horn and Charles R. Johnson Topics in matrix analysis (Cambridge University Press, 1991). 228.

8. Rudolf Kippenhahn, Über den Wertevorrat einer Matrix, Math. Nachr. 6 (1951), 193

9. Allen Knudson and Terrance Tao, Honeycombs and sums of Hermitian matrices, Notices Amer. Math. Soc., 49 (2) (Feb. 2001), 175-186.

10. John S. Lancaster, The boundary of the numerical range, Proc. Amer. Math. Soc., 49, (1975), 393-398.

11. Chi-Kwong Li, Chen-Han Sung and Nam-Kiu Tsing, $c$-Convex matrices: Characterizations, inclusion relations and normality, Linear and Multilinear Algebra 25 (1989), 275-287.

12. Marvin Marcus, Benjamin N. Moyals and Ivan Filippenko, Normality and the higher numerical range, Canad. J. Math., 30 (1978), p419-430.

13. Marvin Marcus and Ivan Filippenko, Nondifferentiable points on the boundary of the numerical range, Linear Algebra Appl., 21 (1978), no.3, 217-232.

14. R. Tyrrell Rockafellar, Convex analysis, (Princeton University Press, 1970).

15. O. Toeplitz, Das algebraische Analogon zu einem Satz von Fejér, Math. Z. 2 (1918), 187-197.

16. Roger Webster, Convexity (Oxford University Press, 1994). 